\title{
LETTERS
}

\section{Assessing methodologic quality of a meta-analysis on managing chronic disease in older adults}

I read with great interest the recent article by Kastner and colleagues on the effectiveness of multimorbidity interventions for older adults with chronic diseases. ${ }^{1}$

I assessed the study's methodological quality using the 16-item AMSTAR 2 (Assessing the Methodological Quality of Systematic Reviews) appraisal tool. ${ }^{2}$ The study scored 12 items out of 16 on AMSTAR 2 and lost points from items 12 and 13 (related to issues of risk of bias), 14 (related to heterogeneity) and 15 (related to publication bias).

Risk of bias was well assessed by appropriate tools, but the findings of this assessment were not accounted for in the analysis through meta-regression or subgroup analysis. The Interpretation section included no discussion on the likely impact of risk of bias. Additionally, the fact that no heterogeneity was observed in the study was not mentioned in the discussion. Publication bias is a very important issue and was not assessed with the appropriate statistical tests. A small number of studies included to estimate the pooled effect size in some subgroups may be the reason for this omission.
To evaluate clinical significance, the prediction interval has been proposed instead of the use of confidence intervals to denote statistical significance. ${ }^{3}$ I suggest that the authors calculate the prediction interval for evaluating clinical significance. ${ }^{3}$

This was a well-written paper and most items were followed appropriately; noncritical weaknesses (e.g., 12, 14) could be ignored, but items 13 and 15 should be taken into account. My AMSTAR 2 assessment shows that this study would be classified as "low" quality.

\section{References}

1. Kastner M, Cardoso R, Lai Y, et al. Effectiveness of interventions for managing multiple high-burden chronic diseases in older adults: a systematic review and meta-analysis. CMAJ 2018;190:E1004.

2. Shea BJ, Reeves BC, Wells G, et al. AMSTAR 2: a critical appraisal tool for systematic reviews that include randomised or non-randomised studies of healthcare interventions, or both. BMJ 2017; 358:j4008.

3. Borenstein M, Hedges LV, Higgins JPT, et al. Introduction to meta-analysis. Hoboken (NJ): John Wiley \& Sons; 2009.

Cite as: CMAJ 2019 June 3;191:E617. doi: $10.1503 / \mathrm{cmaj} .71705$

\section{Razieh Bidhendi Yarandi PhD}

Biostatistician, Department of Epidemiology and Biostatistics, School of Public Health, Tehran University of Medical Sciences, Tehran, Iran

Competing interests: None declared. 